\title{
Aberrant Activity in Degenerated Retinas Revealed by Electrical Imaging
}

\author{
Günther Zeck* \\ Neurochip Research Group, Natural and Medical Sciences Institute at the University of Tübingen, Reutlingen, Germany
}

In this review, I present and discuss the current understanding of aberrant electrical activity found in the ganglion cell layer (GCL) of rod-degenerated $(r d)$ mouse retinas. The reported electrophysiological properties revealed by electrical imaging using highdensity microelectrode arrays can be subdivided between spiking activity originating from retinal ganglion cells (RGCs) and local field potentials (LFPS) reflecting strong transmembrane currents within the GCL. RGCs in rd retinas show increased and rhythmic spiking compared to age-matched wild-type retinas. Fundamental spiking frequencies range from 5 to $15 \mathrm{~Hz}$ in various mouse models. The rhythmic RGC spiking is driven by a presynaptic network comprising All amacrine and bipolar cells. In the healthy retina this rhythm-generating circuit is inhibited by photoreceptor input. A unique physiological feature of $r d$ retinas is rhythmic LFP manifested as spatially-restricted low-frequency (5-15 Hz) voltage changes. Their spatiotemporal characterization revealed propagation and correlation with RGC spiking. LFPs rely on gap-junctional coupling and are shaped by glycinergic and by GABAergic transmission. The aberrant RGC spiking and LFPs provide a simple readout of the functionality of the remaining retinal circuitry which can

OPEN ACCESS

Edited by:

Steven F. Stasheff,

University of lowa, USA

Reviewed by: Botir T. Sagdullaev, Weill Cornell Medical College, USA Yongsook Goo,

Chungbuk National University School of Medicine, South Korea

*Correspondence: Günther Zeck guenther.zeck@nmi.de

Received: 15 November 2015 Accepted: 25 January 2016 Published: 08 February 2016

Citation: Zeck G (2016) Aberrant Activity in Degenerated Retinas Revealed by Electrical Imaging.

Front. Cell. Neurosci. 10:25. doi: 10.3389/fncel.2016.00025 be used in the development of improved vision restoration strategies.

Keywords: ganglion cells, rod-degeneration, microelectrode array, extracellular recording, mouse retina

\section{MOTIVATION: ELECTRICAL IMAGING OF A RETINAL CELL LAYER}

Imaging the electrical activity in three-dimensional neuronal tissue such as the retina relies on sensors which monitor changes of the transmembrane potential or of intracellular ion concentrations. The generation of action potentials leads to changes of intracellular calcium, which are revealed by fluorescence imaging of calcium indicators. For an introduction to calcium imaging in the retina and a comparison with electrical recording see the literature (Briggman and Euler, 2011). Changes of the transmembrane potential and associated transmembrane currents during action potentials or sub-threshold membrane fluctuations modulate the extracellular potential, which is recorded by extracellular electrodes (Fromherz, 2002; Stett et al., 2003). High-frequency $(\sim 1 \mathrm{kHz})$ changes of the extracellular potentials are assigned to action potentials, while lowfrequency changes $(5-100 \mathrm{~Hz}$ ) are assigned to field potentials (Buzsáki et al., 2012; Einevoll et al., 2013).

In retina research, the most studied retinal layer from a physiological perspective is the ganglion cell layer (GCL), due to its easy accessibility in ex vivo preparations. Throughout this review, I refer to simultaneous electrical recording by thousands of micrometer-sized electrodes with high spatial $(\sim 10-30 \mu \mathrm{m})$ and temporal resolution $(\sim 20 \mu \mathrm{s})$ as electrical imaging, 
in accordance with previous studies (Zeck et al., 2011; Greschner et al., 2014). The specific ganglion cell spike patterns generate one type of "electrical image" of the retinal ganglion cell layer. A second type of "electrical images" is obtained by investigating field potentials in the low frequency range. In the following sections, I will review our current knowledge of these two physiological properties (spiking and LFPs) in the GCL of different rod-degenerated $(r d)$ mouse retinas.

\section{ELECTRICAL IMAGING OF SPIKING ACTIVITY IN THE GANGLION CELL LAYER}

Among the first results revealed by electrical imaging of the GCL was the wave-like spiking activity ("retinal wave") in the developing retina (Meister et al., 1991). This activity reflects near-synchronous ganglion cell activity over several millimeters, separated by second-long periods of inactivity. Recently, the development of high-density CMOS-based MEAs comprising several thousand electrodes densely packed in a few square millimeters (Eversmann et al., 2003; Heer et al., 2007; Berdondini et al., 2009) has enabled imaging of retinal waves and their propagation patterns with unprecedented spatial and temporal resolution (Maccione et al., 2014), revealing their shrinkage during ontogeny to the size of the spatial receptive fields of retinal ganglion cells (RGCs).

In the healthy and mature retinas, wave-like activity ceases under physiological conditions. Two important findings revealed by simultaneous electrical recording from multiple ganglion cells are relevant for the understanding of RGC activity in photoreceptor-degenerated retinas: (i) Analysis of pair-wise RGC activity demonstrated functional coupling driven by either reciprocal/gap-junctional coupling between RGCs, by common input from one presynaptic bipolar cell, or by a combination of both modalities (Brivanlou et al., 1998). Correlated activity decreases with developmental age (Demas et al., 2003) but is maintained throughout adulthood for certain cell types. The coupling strength is light dependent (Hu et al., 2010). Electrical coupling between OFF alpha-like RGCs but not between ON alpha-like RGCs has been reported (Hu et al., 2010). Electrical coupling synchronizes the activity of neighboring RGCs, while cells of different polarity (ON vs. OFF RGCs) show phase-shifted activity. (ii) Secondly, the disruption of photoreceptor input achieved by pharmacological intervention or by bleaching of the retina unmasked spontaneous rhythmic activity in RGCs, as revealed by calcium imaging (Toychiev et al., 2013b), patch clamp recording (Toychiev et al., 2013b) or by electrical imaging using CMOS-MEAs (Menzler et al., 2014). This result suggests that rhythmic RGC activity in the mature retina may be triggered by the missing photoreceptor input.

Indeed, in the photoreceptor-degenerated $r d 1$ retina, extracellular recordings from large populations of RGCs measured increased spontaneous activity as compared to the activity in age-matched wild-type retinas (Ye and Goo, 2007; Stasheff, 2008), revealing hyperactivity and for some cells rhythmic activity. The result of elevated and rhythmic ganglion cell spiking has been confirmed and extended by many others, including recordings with CMOS MEAs (Menzler and Zeck, 2011; Menzler et al., 2014) or patch clamp pipettes (Margolis et al., 2008; Borowska et al., 2011; Yee et al., 2012). Ganglion cell hyperactivity and rhythmicity has been reported in a second $r d$ mouse strain (rd10; Goo et al., 2011; Stasheff et al., 2011; Biswas et al., 2014; Menzler et al., 2014) as well as in mouse models in which synaptic transmission between photoreceptors and bipolar cells is dysfunctional $\left(\mathrm{Crx}^{-/-}\right.$and nob mouse; Demas et al., 2006; Soto et al., 2012). The two measures, hyperactivity and rhythmicity are distinct quantities. Hyperactivity is evaluated as the mean number of spikes counted over a time period of several seconds. Rhythmicity is revealed as a peak in the power spectra of the transmembrane currents (Yee et al., 2012) or in the spike-train autocorrelograms (Stasheff, 2008; Menzler and Zeck, 2011). RGC hyperactivity has been detected in $r d 1$ and in $r d 10$ when compared to wild-type retinas recorded under similar experimental conditions. The absolute spike rate values differ between the studies, with lower spike rates when ACSF was used as a recording medium (Ye and Goo, 2007; Stasheff, 2008; Goo et al., 2011; Stasheff et al., 2011) and higher values with Ames' medium (Menzler and Zeck, 2011; Margolis et al., 2014; Ivanova et al., 2015). The spiking rhythmicity reported in these studies exhibits a maximum in the range of $4-15 \mathrm{~Hz}$ in all studied retinas. This parameter appears to be independent of experimental conditions (recording buffer, temperature range) but varies between strains. The oscillatory ganglion cell activity found in $r d 1$ RGC exhibits the highest frequency $(\sim 10 \mathrm{~Hz})$, while this value declines for rd10 $(5-7 \mathrm{~Hz})$ or nob mice $(4 \mathrm{~Hz})$. However, the reported rhythmicity is not seen in all RGCs and appears to vary over time (Goo et al., 2011; Biswas et al., 2014). Rhythmic RGC activity therefore needs to be understood as an intrinsic property of photoreceptor-degenerated or of photoreceptor-deficient retinas (see explanation below) which occurs to different degrees across the GCL.

\section{Mechanism of Rhythm Generation and its Implications for RGC Activity}

The cellular origin of the rhythmic ganglion cell activity has been reviewed in detail (Trenholm and Awatramani, 2015). Briefly, AII amacrine cells are thought to act as rhythm generators. They display rhythmic transmembrane voltage oscillations of $\sim 10 \mathrm{~Hz}$ when isolated from synaptically coupled cells by pharmacological blockers (Choi et al., 2014; Margolis et al., 2014). An alternative explanation suggests the rhythm may be generated in the electrically-coupled network of ON bipolar-AII amacrine cell (Trenholm et al., 2012). In both models the interplay between fast activating sodium channels and slower potassium channels (M-channels) in hyperpolarized AII cells evokes the rhythm generation. Although a quantitative evaluation of the different oscillatory frequencies $(4-15 \mathrm{~Hz}$ among strains, detailed in the previous section) is missing, the increased rhythmic frequency of AII transmembrane voltage for depolarized midpoint membrane potentials (Choi et al., 2014) indicates a pure biophysical mechanism. In addition to the AII amacrine-bipolar cell rhythm generator, a second 
rhythmic network in the outer retina which shows stronger degeneration has been reported and reviewed by Euler and Schubert (2015).

The rhythm generation by the AII amacrine-ON bipolar cell network predicts a phase-shifted activity in $\mathrm{ON}$ and OFF RGCs. OFF bipolar cells, which excite the OFF RGCs, receive glycinergic input from AII amacrine cells while $\mathrm{ON}$ bipolar cells, which excite ON RGCs, are connected to AII amacrine cells by gap junctions. Indeed, phase-shifted activity has been recorded in double-patch clamp experiments in $r d 1$ for nearby alpha-like cell pairs of opposite polarity whereas there was no phase shift between alpha-like RGCs of the same polarity (Margolis et al., 2008). However, random phaseshifted activity between nearby RGC pairs (separation $<200 \mu \mathrm{m}$ ) has been inferred from spike-train cross-correlation analysis of unspecified cell types (Menzler et al., 2014). This result strongly suggests that additional neurons, synaptically connected to RGCs, are rhythmic in $r d 1$ retinas (Margolis et al., 2014). In contrast, in wild-type retinas, where rhythmic activity in RGCs was induced by suppressing the photoreceptor input (Menzler et al., 2014), the phase shifted spiking of nearby RGCs was either zero or in a relatively narrow range of $60-90^{\circ}$. A similar result was found for a small number of rhythmic rd10 RGCs.

In conclusion, the phase-shifted RGC activity predicted by the current model of rhythm generation has been confirmed for alpha-like RGCs in $r d 1$ and for unspecified RGCs in $w t$ and $r d 10$. However, arbitrary phase shifted RGC activity in $r d 1$ indicates that additional neurons in these retinas are rhythmic. The implications of concerted rhythmic activity for a multi-cellular behavior will be discussed in the following section.

\section{LOCAL FIELD POTENTIALS IN THE GANGLION CELL LAYER OF ROD-DEGENERATED RETINAS}

The RGC spiking properties do not provide a complete description of the functional changes occurring in $r d$ retinas. Patch clamp recordings reveal strong sub-threshold oscillations of transmembrane potential (Choi et al., 2014) with frequencies in the same range as the spiking activity. Voltage clamp recordings at different holding potentials demonstrated that the excitatory and inhibitory input currents are rhythmic-although to a different degree in different cell types (Margolis et al., 2008; Yee et al., 2014). These single cell properties translate at a global level to low-frequency field potentials as revealed by MEA recordings. The changes of the extracellular voltage caused by correlated transmembrane currents are recorded as local field potentials (LFPs), given that they are spatially restricted within millisecond-long time intervals. LFPs have been electrically imaged in various brain areas, such as the well-known propagation along the tri-synaptic hippocampal formation (Ferrea et al., 2012) or in cortical structures (Viventi et al., 2011) revealing epileptiform activities. In the healthy and pharmacologically unperturbed mammalian retina LFPs have never been reported. This is most probably attributed to the small local circuits projecting to different types of ganglion cell and to the time-balanced excitatory and inhibitory input. LFPs occur at a time scale of tens of milliseconds,

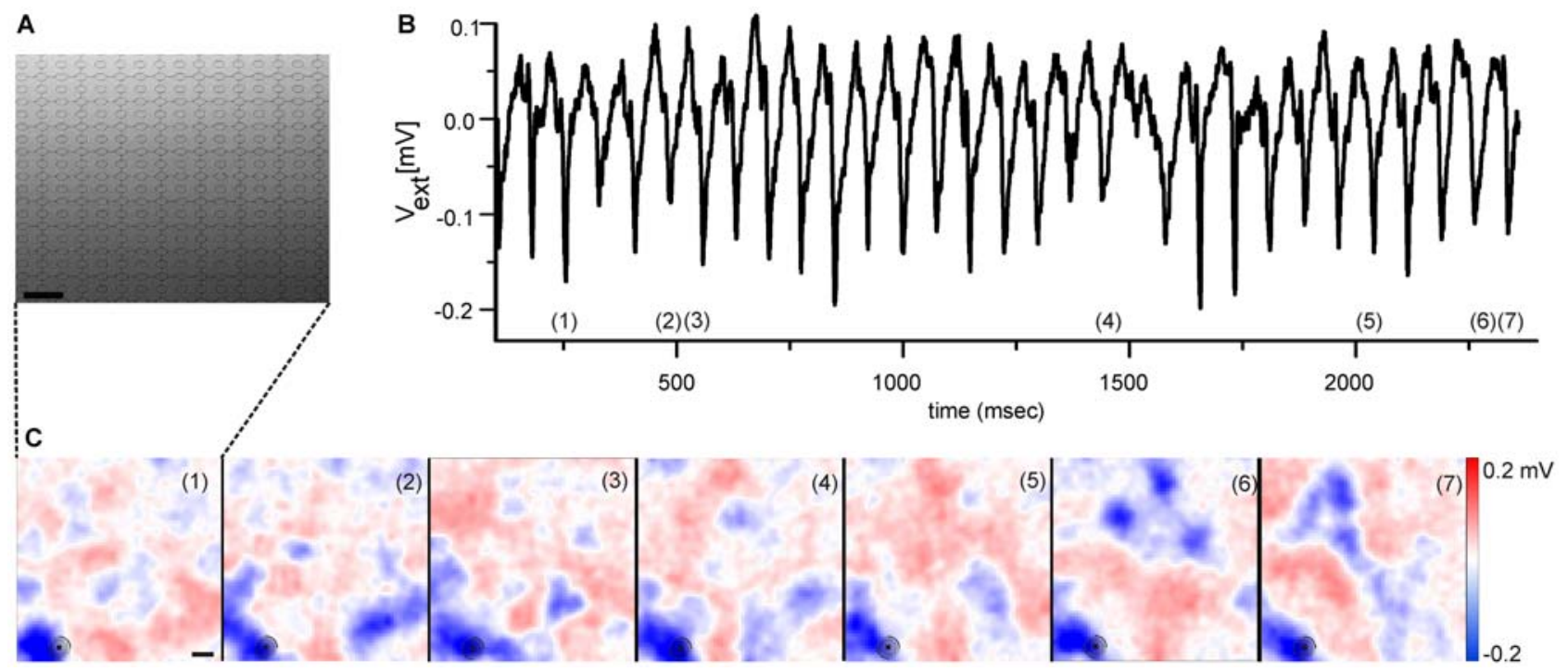

FIGURE 1 | Electrical imaging the rhythmic local field potentials (LFPs) in the ganglion cell layer (GCL) using high-density microelectrode arrays. (A) Raster electron micrograph displaying part of a high-density microelectrode array. Round structures represent the recording electrodes. Scale bar: $32 \mu \mathrm{m}$. Figure modified from Bertotti et al. (2014). (B) Extracellular voltage trace recorded by one selected electrode [marked with circle in the lower left corner of each image in (C)] reveals the rhythmically occurring LFP. The voltage trace has been low-pass filtered $(<100 \mathrm{~Hz})$. (C) Selected color coded images of the extracellular voltage recorded by a high-density microelectrode array as shown in (A). The numbers in each image correspond to numbers in (B), which identify the time of maximal LFP amplitude. The periodically occurring LFPs share a high spatial similarity. Scale bar: $100 \mu \mathrm{m}$. (B,C) modified from Menzler and Zeck (2011). 
whereas excitation and inhibition are balanced within a few milliseconds.

In rod-photoreceptor degenerated mouse retinas ( $r d 1$ and rd10) low-frequency extracellular voltage changes have been reported by different labs (Ye and Goo, 2007; Goo et al., 2011; Menzler and Zeck, 2011; Biswas et al., 2014; Menzler et al., 2014). The LFP frequencies are of the same value as the rhythmic spiking observed in these retinas, i.e., 5-15 Hz. Moreover, LFP maxima and spiking often correlate without time lags (Menzler and Zeck, 2011). This finding together with the observation that LFPs exist without spiking RGCs was an early indicator that rhythmic presynaptic activity drives the aberrant RGC spiking.

A quantitative analysis of the detected LFP size is inherently difficult, as they develop over time in size and amplitude. However, two qualitative LFP features are worth noting. Their size at maximal amplitude extends over an area of $\sim 200 \mu \mathrm{m}$ (see Figure $\mathbf{1}$ and Menzler and Zeck, 2011) suggesting that only a few cells contribute to the initiation of the rhythm. Secondly, LFPs show mostly negative extracellular voltages (blue colors in Figure 1C), indicating strong inward currents. The outward currents are spatially more widely distributed, reflected in the shallower red colors seen in Figure 1C. Similar findings can be inferred from the studies by Menzler and Zeck (2011), Biswas et al. (2014) and correspond to the strong inward currents reported in Yee et al. (2012). A quantitative evaluation which relates the LFPs through a current source density analysis to the generating cells and cellular compartments (Ness et al., 2015) has not been performed so far.

As for the spiking properties, spatial electrical imaging the LFPs reveals qualitative differences between $r d 1$ and $r d 10$ retinas. In $r d 1$ retinas the "pulsatile" LFPs propagate at a median speed of $8 \mathrm{~mm} / \mathrm{sec}$ (Menzler and Zeck, 2011). In the $r d 10$ GCL it is unclear if LFP propagation occurs (Menzler et al., 2014). The putative propagation reported in a previous study by Biswas et al. (2014) is somewhat difficult to interpret given the large spacing between electrodes. Propagation was suggested to depend on electrical coupling; however there is no conclusive experimental evidence transforming propagating LFPs into stationary ones. Furthermore, the repetitive occurrence of LFPs in the same retinal portion (see Figure 1) and evidence provided by Menzler and Zeck (2011), indicates a considerable spatial variability and LFP dynamics which requires a thorough quantitative analysis in future studies.

The current knowledge about the retinal neurons involved in the generation of rhythmic activity and the corresponding functional readout using electrical imaging is summarized in Figure 2. This functional characterization is based on available methodology; including appropriate filtering (low-pass and highpass), spike and LFP identification, spike sorting, computation of auto- and cross-correlograms and calculation of power spectral densities. In addition to the aberrant, rhythmic activity detected in photoreceptor-degenerated retinas one should remember that a considerable percentage of RGCs show normal activity, most probably in areas of the GCL with less circuit degeneration.
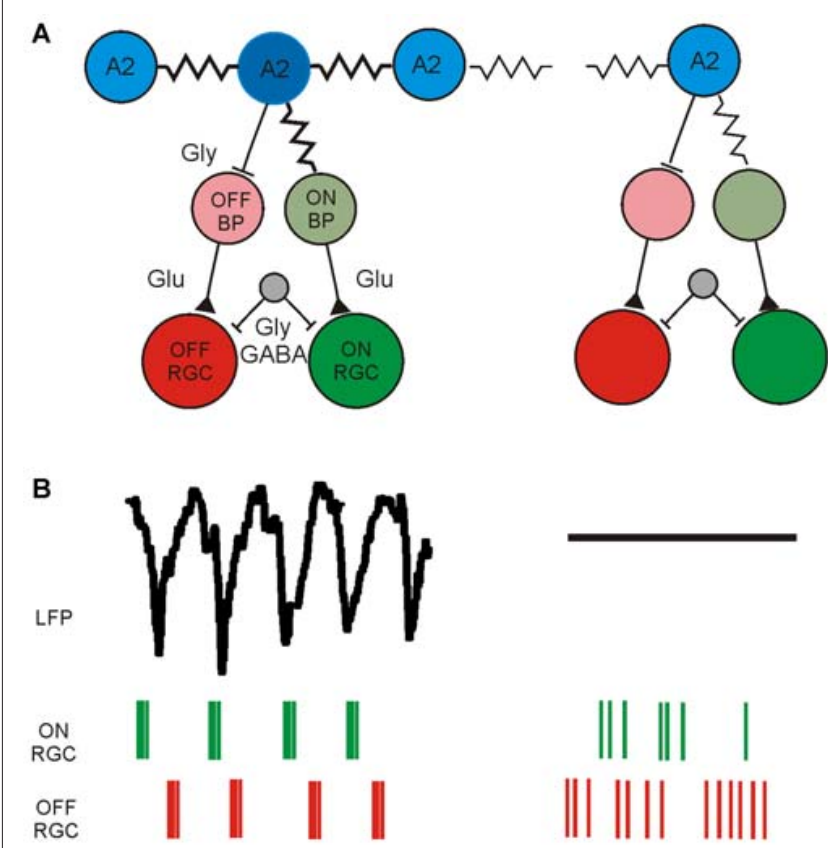

FIGURE 2 | Schematic diagram of synaptically connected retinal neurons generating rhythmic activity in the GCL of photoreceptor-degenerated retinas in the low and high frequency range. (A) Simplified schematic synaptic connectivity in rd retinas. The rhythmic activity is generated and spreads in the electrically coupled network of All amacrine cells and ON cone-bipolar cells (ON CB) and propagates through glutamatergic excitation to ON-type RGCs. Through glycinergic inhibition the rhythmic activity reaches OFF RGCs. An unidentified cell (gray) modulates the rhythmic spiking through GABAergic and/or glycinergic input. The rhythmic activity does not occur over the entire retina, symbolized in the weakly coupled network on the right. Part of the figure modified from (Margolis et al., 2014). (B) Low-frequency LFP and rhythmic, high-frequency spiking in two different retinal ganglion cell types (ON RGC and OFF RGC) driven by the rhythmic presynaptic network. Rhythmic LFPs and RGC spiking is probably recorded only across strongly coupled areas of the RGC layer, whereas in weakly coupled regions the LFP size decreases and the spiking becomes arrhythmic.

In the following last section, I discuss how aberrant ganglion cell activity and the spatio-temporal LFP dynamics affect strategies for visual restoration in $r d$ retinas.

\section{ADVANCES AND CURRENT LIMITATIONS OF VISUAL RESTORATION IN ROD-DEGENERATED RETINAS}

A prerequisite for visual restoration in patients who are blind due to loss of photoreceptors is the survival of inner retinal neurons and their synaptic connections to higher visual areas. In $r d$ mouse models previous work has revealed changes of bipolar cell morphology (Strettoi et al., 2002; Gargini et al., 2007; Barhoum et al., 2008) but a well preserved RGC morphology (Mazzoni et al., 2008) and mosaic-like arrangement of at least two RGC types (Lin and Peng, 2013). Furthermore, RGCs in $r d 1$ and in $r d 10$ retina retain their synaptic connections with higher visual centers (Bi et al., 2006; 
Lagali et al., 2008; Lin et al., 2008; Ivanova et al., 2015). Specifically, the aberrant RGC activity propagates to higher brain areas and may hinder visual coding (Ivanova et al., 2015).

Strategies for restoration of vision may therefore address the following questions, assuming that the lessons learned in blind mice translate to the human case. How can the aberrant ganglion cell activity be overcome by external stimuli and to what degree do external stimuli evoke near-physiological RGC response patterns?

The first question has been tackled by employing pharmacological intervention (Toychiev et al., 2013a; Barrett et al., 2015). For a certain number of RGCs and a relatively early degeneration age the application of gap junction blockers reduced hyperactivity and at the same time enabled RGC activation either by electrical stimulation (Toychiev et al., 2013a) or by optogenetic cell transfection followed by light stimulation (Barrett et al., 2015). While the reduction of gap-junctional coupling might be beneficial for approaches targeting the RGCs directly, such as epiretinal electric stimulation (Chuang et al., 2014) or optogenetic activation of ChR2 expressing RGCs (Bi et al., 2006), it might corrupt visual processing in strategies targeting the inner retinal circuitry, such as subretinal electrical stimulation (Zrenner et al., 2011) or optogenetic intervention at the bipolar cell level (Lagali et al., 2008; van Wyk et al., 2015). Although MEAs have been used to detect evoked RGC activity (Barrett et al., 2015), no electrical imaging of the GCL has been performed.

The second question of how close the artificially-evoked responses in blind retinas resemble light-activated activity in healthy retinas has been addressed for electrical (O'Hearn et al., 2006; Ryu et al., 2010; Goetz et al., 2015; Lorach et al., 2015) and for optogenetic stimulation (Busskamp et al., 2010; van Wyk et al., 2015). Subretinal electric stimulation of dystrophic rat retina evoked spatially selective RGC response patterns which were of the same size as light-evoked receptive fields in healthy retinas (Lorach et al., 2015). This remarkable result was achieved by recording the evoked RGC response using a high-density MEA. However, the stimulation method (spatial white noise) used to map the electrically evoked receptive fields did not reveal putative rhythmic RGC activity, as reported in

\section{REFERENCES}

Barhoum, R., Martínez-Navarrete, G., Corrochano, S., Germain, F., FernandezSanchez, L., de la Rosa, E. J., et al. (2008). Functional and structural modifications during retinal degeneration in the $r d 10$ mouse. Neuroscience 155 , 698-713. doi: 10.1016/j.neuroscience.2008.06.042

Barrett, J. M., Degenaar, P., and Sernagor, E. (2015). Blockade of pathological retinal ganglion cell hyperactivity improves optogenetically evoked light responses in rd1 mice. Front. Cell. Neurosci. 9:330. doi: 10.3389/fncel.2015. 00330

Berdondini, L., Imfeld, K., Maccione, A., Tedesco, M., Neukom, S., KoudelkaHep, M., et al. (2009). Active pixel sensor array for high spatiotemporal resolution electrophysiological recordings from single cell to large scale neuronal networks. Lab Chip 9, 2644-2651. doi: 10.1039/b90 $7394 \mathrm{a}$ another study (Ryu et al., 2010) for pulsatile stimulation of rd1 RGCs. Common to the electrical stimulation approaches in blind retinas is the very small percentage of activated OFF RGC responses (Goetz et al., 2015). Therefore light activation of optogenetically transduced halorhodopsin in remaining cones (Busskamp et al., 2010) or activation of a chimeric melanopsinmGluR6 molecule in rd1 ON bipolar cells (van Wyk et al., 2015) is attractive, as it restored ON and OFF RGC response polarities in recorded RGCs. For optogenetic stimulation little is known so far about the distortion by aberrant RGC or LFP activity, since the above-cited studies relied on single cell recordings.

In future, electrical imaging of the retinal GCL may improve future vision strategies in two ways. First, it can indicate if vision restoration is successful by comparing the artificially induced RGC patterns to physiological patterns. Several of the above cited studies performed a basic characterization of the induced activity, which can easily be extended to a comprehensive electrophysiological picture. Second, electrical imaging and analysis of the RGC population activity in blind retinas may reveal to what degree the aberrant rhythmicity impairs the coding of artificially evoked responses. Studies in frog and in cat retinas indicate a beneficial effect of oscillatory retinal activity for visual coding (Ishikane et al., 2005; Koepsell et al., 2009) while a recent study using blind retina underlines the distortion introduced by the aberrant activity (Ivanova et al., 2015).

\section{AUTHOR CONTRIBUTIONS}

GZ wrote the manuscript.

\section{FUNDING}

Funding of this work was in part obtained from the German Federal Ministry of Education and Research through grant no. 1312038.

\section{ACKNOWLEDGMENTS}

GZ thanks Peter Jones for English editing of the manuscript.

Bertotti, G., Velychko, D., Dodel, N., Keil, S., Wolansky, D., Tillak, B., et al (2014). "A CMOS-based sensor arrayfor in-vitro neural tissue interfacing with 4225 recording sites and 1024 stimulation sites," in Biomedical Circuits and Systems Conference (BioCAS), (Lausanne: IEEE), 304-307. doi: 10.1109/BIOCAS.2014.6981723

Bi, A. D., Cui, J. J., Ma, Y. P., Olshevskaya, E., Pu, M. L., Dizhoor, A. M., et al. (2006). Ectopic expression of a microbial-type rhodopsin restores visual responses in mice with photoreceptor degeneration. Neuron 50, 23-33. doi: 10. 1016/j.neuron.2006.02.026

Biswas, S., Haselier, C., Mataruga, A., Thumann, G., Walter, P., and Müller, F. (2014). Pharmacological analysis of intrinsic neuronal oscillations in $r d 10$ retina. PLoS One 9:e99075. doi: 10.1371/journal.pone.0099075

Borowska, J., Trenholm, S., and Awatramani, G. B. (2011). An intrinsic neural oscillator in the degenerating mouse retina. J. Neurosci. 31, 5000-5012. doi: 10 1523/jneurosci.5800-10.2011 
Briggman, K. L., and Euler, T. (2011). Bulk electroporation and population calcium imaging in the adult mammalian retina. J. Neurophysiol. 105, 2601-2609. doi: 10.1152/jn.00722.2010

Brivanlou, I. H., Warland, D. K., and Meister, M. (1998). Mechanisms of concerted firing among retinal ganglion cells. Neuron 20, 527-539. doi: 10.1016/s08966273(00)80992-7

Busskamp, V., Duebel, J., Balya, D., Fradot, M., Viney, T. J., Siegert, S., et al. (2010). Genetic reactivation of cone photoreceptors restores visual responses in retinitis pigmentosa. Science 329, 413-417. doi: 10.1126/science.1190897

Buzsáki, G., Anastassiou, C. A., and Koch, C. (2012). The origin of extracellular fields and currents-EEG, ECoG, LFP and spikes. Nat. Rev. Neurosci. 13, 407-420. doi: 10.1038/nrn3241

Choi, H., Zhang, L., Cembrowski, M. S., Sabottke, C. F., Markowitz, A. L., Butts, D. A., et al. (2014). Intrinsic bursting of AII amacrine cells underlies oscillations in the $r d 1$ mouse retina. J. Neurophysiol. 112, 1491-1504. doi: 10.1152/jn.00437. 2014

Chuang, A. T., Margo, C. E., and Greenberg, P. B. (2014). Retinal implants: a systematic review. Br. J. Ophthalmol. 98, 852-856. doi: 10.1136/bjophthalmol2013-303708

Demas, J., Eglen, S. J., and Wong, R. O. L. (2003). Developmental loss of synchronous spontaneous activity in the mouse retina is independent of visual experience. J. Neurosci. 23, 2851-2860.

Demas, J., Sagdullaev, B. T., Green, E., Jaubert-Miazza, L., McCall, M. A., Gregg, R. G., et al. (2006). Failure to maintain eye-specific segregation in nob, a mutant with abnormally patterned retinal activity. Neuron $50,247-259$. doi: 10.1016/j. neuron.2006.03.033

Einevoll, G. T., Kayser, C., Logothetis, N. K., and Panzeri, S. (2013). Modelling and analysis of local field potentials for studying the function of cortical circuits. Nat. Rev. Neurosci. 14, 770-785. doi: 10.1038/nrn3599

Euler, T., and Schubert, T. (2015). Multiple independent oscillatory networks in the degenerating retina. Front. Cell. Neurosci. 9:444. doi: 10.3389/fncel.2015. 00444

Eversmann, B., Jenkner, M., Hofmann, F., Paulus, C., Brederlow, R., Holzapfl, B., et al. (2003). A $128 \times 128$ CMOS biosensor array for extracellular recording of neural activity. IEEE J. Solid-State Circuits 38, 2306-2317. doi: 10.1109/jssc. 2003.819174

Ferrea, E., Maccione, A., Medrihan, L., Nieus, T., Ghezzi, D., Baldelli, P., et al. (2012). Large-scale, high-resolution electrophysiological imaging of field potentials in brain slices with microelectronic multielectrode arrays. Front. Neural Circuits 6:80. doi: 10.3389/fncir.2012.00080

Fromherz, P. (2002). Electrical interfacing of nerve cells and semiconductor chips. Chemphyschem 3, 276-284. doi: 10.1002/1439-7641(20020315)3:3<276::aidcphc276>3.0.co;2-a

Gargini, C., Terzibasi, E., Mazzoni, F., and Strettoi, E. (2007). Retinal organization in the retinal degeneration $10(r d 10)$ mutant mouse: a morphological and ERG study. J. Comp. Neurol. 500, 222-238. doi: 10.1002/cne.21144

Goetz, G., Smith, R., Lei, X., Galambos, L., Kamins, T., Mathieson, K., et al. (2015). Contrast sensitivity with a subretinal prosthesis and implications for efficient delivery of visual information. Invest. Ophthalmol. Vis. Sci. 56, 7186-7194. doi: 10.1167 /iovs.15-17566

Goo, Y. S., Ahn, K. N., Song, Y. J., Ahn, S. H., Han, S. K., Ryu, S. B., et al. (2011). Spontaneous Oscillatory rhythm in retinal activities of two retinal degeneration ( $r d 1$ and $r d 10)$ mice. Korean J. Physiol. Pharmacol. 15, 415-422. doi: 10.4196/kjpp.2011.15.6.415

Greschner, M., Field, G. D., Li, P. H., Schiff, M. L., Gauthier, J. L., Ahn, D., et al. (2014). A polyaxonal amacrine cell population in the primate retina. J. Neurosci. 34, 3597-3606. doi: 10.1523/jneurosci.3359-13.2014

Heer, F., Hafizovic, S., Ugniwenko, T., Frey, U., Franks, W., Perriard, E., et al. (2007). Single-chip microelectronic system to interface with living cells. Biosens. Bioelectron. 22, 2546-2553. doi: 10.1016/j.bios.2006.10.003

Hu, E. H., Pan, F., Völgyi, B., and Bloomfield, S. A. (2010). Light increases the gap junctional coupling of retinal ganglion cells. J. Physiol. 588, 4145-4163. doi: 10. 1113/jphysiol.2010.193268

Ishikane, H., Gangi, M., Honda, S., and Tachibana, M. (2005). Synchronized retinal oscillations encode essential information for escape behavior in frogs. Nat. Neurosci. 8, 1087-1095. doi: 10.1038/nn1497

Ivanova, E., Yee, C. W., Baldoni, R. Jr., and Sagdullaev, B. T. (2015). Aberrant activity in retinal degeneration impairs central visual processing and relies on
Cx36-containing gap junctions. Exp. Eye Res. doi: 10.1016/j.exer.2015.05.013 [Epub ahead of print].

Koepsell, K., Wang, X., Vaingankar, V., Wei, Y., Wang, Q., Rathbun, D. L., et al. (2009). Retinal oscillations carry visual information to cortex. Front. Syst. Neurosci. 3:4. doi: 10.3389/neuro.06.004.2009

Lagali, P. S., Balya, D., Awatramani, G. B., Münch, T. A., Kim, D. S., Busskamp, V., et al. (2008). Light-activated channels targeted to ON bipolar cells restore visual function in retinal degeneration. Nat. Neurosci. 11, 667-675. doi: 10.1038/nn. 2117

Lin, B., Koizumi, A., Tanaka, N., Panda, S., and Masland, R. H. (2008). Restoration of visual function in retinal degeneration mice by ectopic expression of melanopsin. Proc. Natl. Acad. Sci. U S A 105, 16009-16014. doi: 10.1073/pnas. 0806114105

Lin, B., and Peng, E. B. (2013). Retinal ganglion cells are resistant to photoreceptor loss in retinal degeneration. PLoS One 8:e68084. doi: 10.1371/journal.pone. 0068084

Lorach, H., Goetz, G., Smith, R., Lei, X., Mandel, Y., Kamins, T., et al. (2015). Photovoltaic restoration of sight with high visual acuity. Nat. Med. 21, 476-482. doi: $10.1038 / \mathrm{nm} .3851$

Maccione, A., Hennig, M. H., Gandolfo, M., Muthmann, O., van Coppenhagen, J., Eglen, S. J., et al. (2014). Following the ontogeny of retinal waves: pan-retinal recordings of population dynamics in the neonatal mouse. J. Physiol. 592, 1545-1563. doi: 10.1113/jphysiol.2013. 262840

Margolis, D. J., Gartland, A. J., Singer, J. H., and Detwiler, P. B. (2014). Network oscillations drive correlated spiking of ON and OFF ganglion cells in the $r d 1$ mouse model of retinal degeneration. PLoS One 9:e86253. doi: 10.1371/journal. pone. 0086253

Margolis, D. J., Newkirk, G., Euler, T., and Detwiler, P. B. (2008). Functional stability of retinal ganglion cells after degeneration-induced changes in synaptic input. J. Neurosci. 28, 6526-6536. doi: 10.1523/jneurosci.1533-08.2008

Mazzoni, F., Novelli, E., and Strettoi, E. (2008). Retinal ganglion cells survive and maintain normal dendritic morphology in a mouse model of inherited photoreceptor degeneration. J. Neurosci. 28, 14282-14292. doi: 10. 1523/jneurosci.4968-08.2008

Meister, M., Wong, R. O. L., Baylor, D. A., and Shatz, C. J. (1991). Synchronous bursts of action potentials in ganglion cells of the developing mammalian retina. Science 252, 939-943. doi: 10.1126/science. 2035024

Menzler, J., Channappa, L., and Zeck, G. (2014). Rhythmic ganglion cella ctivity in bleached and blind adult mouse retina. PLoS One 9:e106047. doi: 10. 1371/journal.pone.0106047

Menzler, J., and Zeck, G. (2011). Network oscillations in rod-degenerated mouse retinas. J. Neurosci. 31, 2280-2291. doi: 10.1523/jneurosci.4238-10.2011

Ness, T. V., Chintaluri, C., Potworowski, J., Łeski, S., Glabska, H., Wójcik, D. K., et al. (2015). Modelling and analysis of electrical potentials recorded in microelectrode arrays (MEAs). Neuroinformatics 13, 403-426. doi: 10. 1007/s12021-015-9265-6

O’Hearn, T. M., Sadda, S. R., Weiland, J. D., Maia, M., Margalit, E., and Humayun, M. S. (2006). Electrical stimulation in normal and retinal degeneration $(r d 1)$ isolated mouse retina. Vision Res. 46, 3198-3204. doi: 10.1016/j.visres.2006. 03.031

Ryu, S. B., Ye, J. H., Goo, Y. S., Kim, C. H., and Kim, K. H. (2010). Temporal response properties of retinal ganglion cells in $r d 1$ mice evoked by amplitudemodulated electrical pulse trains. Invest. Ophthalmol. Vis. Sci. 51, 6762-6769. doi: 10.1167 /iovs.10-5577

Soto, F., Ma, X., Cecil, J. L., Vo, B. Q., Culican, S. M., and Kerschensteiner, D. (2012). Spontaneous activity promotes synapse formation in a cell-typedependent manner in the developing retina. J. Neurosci. 32, 5426-5439. doi: 10. 1523/JNEUROSCI.0194-12.2012

Stasheff, S. F. (2008). Emergence of sustained spontaneous hyperactivity and temporary preservation of OFF responses in ganglion cells of the retinal degeneration (rd1) mouse. J. Neurophysiol. 99, 1408-1421. doi: 10.1152/jn. 00144.2007

Stasheff, S., Shankar, M., and Andrews, M. P. (2011). Developmental time course distinguishes changes in spontaneous and light-evoked retinal ganglion cell activity in $r d 1$ and $r d 10$ mice. J. Neurophysiol. 105, 3002-3009. doi: 10.1152/jn. 00704.2010 
Stett, A., Egert, U., Guenther, E., Hofmann, F., Meyer, T., Nisch, W., et al. (2003). Biological application of microelectrode arrays in drug discovery and basic research. Anal. Bioanal. Chem. 377, 486-495. doi: 10.1007/s00216-003-2149-x

Strettoi, E., Porciatti, V., Falsini, B., Pignatelli, V., and Rossi, C. (2002). Morphological and functional abnormalities in the inner retina of $\mathrm{rd} / \mathrm{rd}$ mouse. J. Neurosci. 22, 5492-5504.

Toychiev, A. H., Ivanova, E., Yee, C. W., and Sagdullaev, B. T. (2013a). Block of gap junctions eliminates aberrant activity and restores light responses during retinal degeneration. J. Neurosci. 33, 13972-13977. doi: 10.1523/JNEUROSCI. 2399-13.2013

Toychiev, A. H., Yee, C. W., and Sagdullaev, B. T. (2013b). Correlated spontaneous activity persists in adult retina and is suppressed by inhibitory inputs. PLoS One 8:e77658. doi: 10.1371/journal.pone.0077658

Trenholm, S., and Awatramani, G. B. (2015). Origins of spontaneous activity in the degenerating retina. Front. Cell. Neurosci. 9:277. doi: 10.3389/fncel.2015. 00277

Trenholm, S., Borowska, J., Zhang, J., Hoggarth, A., Johnson, K., Barnes, S., et al. (2012). Intrinsic oscillatory activity arising within the electrically coupled AII amacrine-ON cone bipolar cell network is driven by voltagegated Na plus channels. J. Physiol. 590, 2501-2517. doi: 10.1113/jphysiol.2011. 225060

van Wyk, M., Pielecka-Fortuna, J., Löwel, S., and Kleinlogel, S. (2015). Restoring the ON switch in blind retinas: opto-mGluR6, a next-generation, cell-tailored optogenetic tool. PLoS Biol. 13:e1002143. doi: 10.1371/journal.pbio.1002143

Viventi, J., Kim, D. H., Vigeland, L., Frechette, E. S., Blanco, J. A., Kim, Y. S., et al. (2011). Flexible, foldable, actively multiplexed, high-density electrode array for mapping brain activity in vivo. Nat. Neurosci. 14, 1599-1605. doi: 10.1038/nn. 2973
Ye, J. H., and Goo, Y. S. (2007). The slow wave component of retinal activity in $\mathrm{rd} / \mathrm{rd}$ mice recorded with a multi-electrode array. Physiol. Meas. 28, 1079-1088. doi: 10.1088/0967-3334/28/9/009

Yee, C. W., Toychiev, A. H., Ivanova, E., and Sagdullaev, B. T. (2014). Aberrant synaptic input to retinal ganglion cells varies with morphology in a mouse model of retinal degeneration. J. Comp. Neurol. 522, 4085-4099. doi: 10. $1002 /$ cne. 23660

Yee, C. W., Toychiev, A. H., and Sagdullaev, B. T. (2012). Network deficiency exacerbates impairment in a mouse model of retinal degeneration. Front. Syst. Neurosci. 6:8. doi: 10.3389/fnsys.2012.00008

Zeck, G., Lambacher, A., and Fromherz, P. (2011). Axonal transmission in the retina introduces a small dispersion of relative timing in the ganglion cell population response. PLoS One 6:e20810. doi: 10.1371/journal.pone.0020810

Zrenner, E., Bartz-Schmidt, K. U., Benav, H., Besch, D., Bruckmann, A., Gabel, V.P., et al. (2011). Subretinal electronic chips allow blind patients to read letters and combine them to words. Proc. Biol. Sci. 278, 1489-1497. doi: 10.1098/rspb. 2010.1747

Conflict of Interest Statement: The author declares that the research was conducted in the absence of any commercial or financial relationships that could be construed as a potential conflict of interest.

Copyright (c) 2016 Zeck. This is an open-access article distributed under the terms of the Creative Commons Attribution License (CC BY). The use, distribution and reproduction in other forums is permitted, provided the original author(s) or licensor are credited and that the original publication in this journal is cited, in accordance with accepted academic practice. No use, distribution or reproduction is permitted which does not comply with these terms. 